\title{
Management of Critical Burn Injuries: Recent Developments
}

\author{
David J. Dries ${ }^{1}$, and John J. Marini ${ }^{2}$ \\ ${ }^{1}$ Department of Surgery and Anesthesiology, ${ }^{2}$ Department of Medicine, University of Minnesota, Minneapolis, MN, USA
}

Background: Burn injury and its subsequent multisystem effects are commonly encountered by acute care practitioners. Resuscitation is the major component of initial burn care and must be managed to restore and preserve vital organ function. Later complications of burn injury are dominated by infection. Burn centers are often called to manage problems related to thermal injury, including lightning and electrical injuries.

Methods: A selected review is provided of key management concepts as well as of recent reports published by the American Burn Association.

Results: The burn-injured patient is easily and frequently over resuscitated, with ensuing complications that include delayed wound healing and respiratory compromise. A feedback protocol designed to limit the occurrence of excessive resuscitation has been proposed, but no new "gold standard" for resuscitation has replaced the venerated Parkland formula. While new medical therapies have been proposed for patients sustaining inhalation injury, a paradigm-shifting standard of medical therapy has not emerged. Renal failure as a specific contributor to adverse outcome in burns has been reinforced by recent data. Of special problems addressed in burn centers, electrical injuries pose multisystem physiologic challenges and do not fit typical scoring systems.

Conclusion: Recent reports emphasize the dangers of over resuscitation in the setting of burn injury. No new medical therapy for inhalation injury has been generally adopted, but new standards for description of burn-related infections have been presented. The value of the burn center in care of the problems of electrical exposure, both manmade and natural, is demonstrated in recent reports.

Key Words: burns; electrical injuries; infection; lightning; resuscitation; smoke inhalation injury.

\section{Introduction}

The burn-injured patient presents special challenges regarding resuscitation requirements, metabolic stress, pattern of complications and determinants of outcome [1]. This review highlights a selected group of papers focused on those aspects of care which are unique to burn centers and the burn-injured patient and contribute in important ways to outcome. This selective review of key principles, recent literature, and summary statements is directed toward the acute care physician or surgeon with an interest in such injuries who does not encounter such problems on a daily basis.

Contemporary discussions of burn resuscitation often feature the Parkland formula proposed by Baxter and coworkers in the 1960s [1,2]. Reviews of recent experience with burn resuscitation suggest that treatment objectives and fluids administered in the approach originally recommended by the Parkland group should be re-examined [3]. What is contemporary thinking about initial fluid administration in the setting of burn injury? The American Burn Association

\footnotetext{
Received on December 2, 2016 Accepted on December 25, 2016

Correspondence to: David J. Dries, Department of Surgery, Regions Hospital, 640 Jackson Street, St. Paul, MN 55101, USA

Tel: +1-651-254-1633, Fax: +1-651-254-1480, E-mail: david.j.dries@healthpartners.com
}

*No potential conflict of interest relevant to this article was reported.

(cc) This is an Open Access article distributed under the terms of the Creative Commons Attribution Non-Commercial License (http://creativecommons.org/ licenses/by-nc/4.0/) which permits unrestricted non-commercial use, distribution, and reproduction in any medium, provided the original work is properly cited. Copyright (c) 2017 The Korean Society of Critical Care Medicine 
(ABA) has recently presented a statement to address this question [4]. The Parkland Burn Center of Dallas, Texas (USA) also published a report on the use of the Parkland formula in the institution where it originated [5]. Apart from understanding the special needs of fluid support, the practitioner should also recognize that sepsis also presents in non-traditional ways in the burn-injured patient [6]. In this paper, we summarize for the non-burn physician and surgeon some of the key aspects of a recent consensus statement produced by the ABA about organspecific infectious complications in the setting of burn injury.

A number of outcome indicators related to burn unit practice are coming into clearer focus. Renal failure has a major impact on mortality in any critical care unit population [7]. We now have data indicating that similar concerns hold true regarding the extensively burned patient. Burn units are often selected to manage other problems of the integument and internal organs that bear resemblance to those of thermal exposure. Lifethreatening lightning strikes and other forms of electrical injury pose problems beyond the degree and pattern of skin exposure. In fact, outcome is generally determined by the degree of internal injury in patients victimized by damaging electrical energy [8]. Common to all varieties of extensive thermal injuries are the challenges to appropriately manage fluid balance and infection hazard.

\section{Resuscitation}

Fluid administration in the setting of burn injury and monitoring of efficacy are addressed by consensus recommendations presented in recent work published in the Journal of Burn Care \& Research [4]. The University of Texas Southwestern group in Dallas also provides a 35 year retrospective and commentary on the present status of the Parkland formula often used to guide fluid therapy in this critical initial stage. This long venerated standard for burn resuscitation has recently been critiqued by multiple studies, and an insightful editorial review pointed out that burn patients frequently receive greater amounts of fluid than predicted [3]. In short, the accuracy and practicality of the original Parkland formula has been questioned. Resolution of these debates is important, in part because many burn centers have 'protocolized' the implementation of the formula by highly trained and specialized nursing personnel (Figure 1).

A valuable retrospective analysis has been conducted of the experience with burn patients treated at Parkland Memorial Hospital Burn Center during a 15 year period from 1991 to 2005 [5]. Included were burns in adults > $19 \%$ total body surface area (TBSA). In this adult group, adequate fluid resuscitation was defined by achieving a urine output of 0.5 to $1.0 \mathrm{~mL} / \mathrm{kg} / \mathrm{hr}$. Over resuscitation was defined as a urine output $>1.0 \mathrm{~mL} / \mathrm{kg} / \mathrm{hr}$. In a review of nearly 500 patients, $43 \%$ received adequate resuscitation based on urine output criteria. Forty-eight percent were over resuscitated. Using these adequacy criteria, however, there was no observed difference in complication rates or mortality incidence between resuscitation categories. Patients were evaluated for inhalation injury with bronchoscopy. Contrary to reports from other centers, the amount of fluid required for adequate resuscitation based on target urine output was not different in patients with inhalation injury as opposed to those without this insult. While other groups have reported [9] that intra-abdominal hypertension and abdominal compartment syndrome commonly occur in burn patients with volume resuscitation in excess of $250 \mathrm{~mL} / \mathrm{kg}$, the Parkland experience indicates only a $1 \%$ incidence of abdominal compartment syndrome, even in burns exceeding $40 \%$ TBSA, where administered resuscitation volumes exceeded $250 \mathrm{~mL} / \mathrm{kg}$. In summary, even in the home of the Parkland formula, actual burn resuscitation frequently does not meet the standard set forth by this clinical strategy. Patients commonly received higher fluid volumes than predicted by the Parkland formula. The Parkland team recommended placing emphasis on calculated formula volumes only as a guide to initial resuscitation, with subsequent fluids titrated to urine output.

A consensus statement has been released from the 
Protocol for Fluid Resuscitation of the Adult Burn Patient:

Begin LR using burn center fluid resuscitation calculations

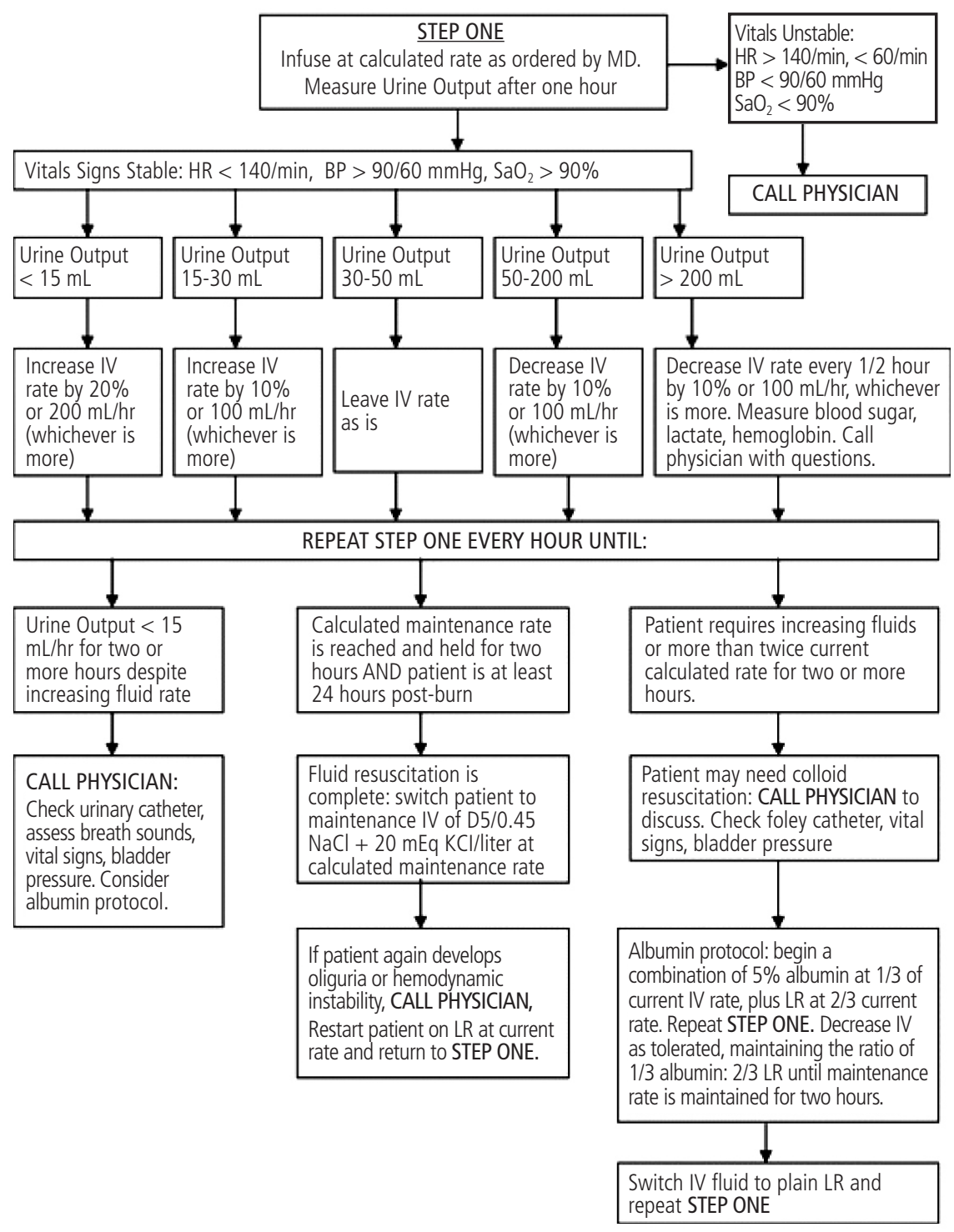

Figure 1. Protocol for fluid resuscitation of adult burn patients. In response to requests from nurses, this protocol was developed to permit nursing staff to manage fluid resuscitation of acute burn patients. Initial fluid rates are calculated by the Parkland formula. Nurses begin hourly infusion, measure urine output, and adjust fluids according to patient response. Development of unstable vital signs, inadequate response to fluids, or persistently high fluid requirements prompt a call to the physician. A pathway to begin colloid replacement exists for patients who display increasing fluid requirements or develop symptoms of torso compartment syndrome. Adopted from references [3] with permission. LR: lactated Ringer's solution; D5: 5\% dextrose.
ABA regarding burn/shock resuscitation [4]. Notably, no "standards" for the approach to the resuscitation of burninjured patients have been derived from high quality contemporary studies. However, a number of "guidelines" are supported by evidence of lesser strength. "Guidelines" and "Options" from the ABA are listed in the Journal of Burn Care \& Research [4].

Lacking unequivocal evidence, there currently is little agreement regarding optimal fluid composition, rate of fluid administration, and the role of colloids, such as al- bumin. No resuscitation parameters geared to regulating individual patient fluid needs by formula perform clearly better than routine hemodynamic endpoints and adequate urine output. Practitioners must be compulsive in providing adequate fluids but must also avoid excessive resuscitation. Many centers begin with the Parkland formula, which has now been renamed the Consensus formula for resucitation because it is the most widely used approach. This formula provides $4 \mathrm{~mL} / \mathrm{kg} / \%$ TBSA burned, describing the amount of lactated Ringers solution required 
in the first 24 hours after burn injury where ' $\mathrm{kg}$ ' represents initial patient weight, and ' $\%$ TBSA' is the extent of 2nd and 3rd degree burn injury. Starting from the time of burn injury, half of the fluid is given in the first 8 hours and the remaining half is given over the next 16 hours. Unfortunately, rapid determination of \% TBSA burn and calculation of fluid requirements can be difficult and is often incorrect when the clinician charged with treating burns is relatively inexperienced. Many centers are evolving resuscitation protocols such as that given in Figure 1 from the University of Utah. Fluid administration in this protocol begins with the Consensus formula, and fluids are titrated based on patient response [1].

Three additional points of clarification regarding burn resuscitation should be made. First, many patients, particularly those with burns $<20 \%$ TBSA, may be candidates for oral resuscitation as an intact gastrointestinal tract tolerates large amounts of administered fluid. Enteral resuscitation should be considered when resources are limited, an austere setting is encountered, and the patient is able to tolerate enteral intake. Second, invasive hemodynamic monitors including central venous catheters and pulmonary artery catheters have been employed to optimize burn resuscitation in a variety of prospective and retrospective studies. Patients with invasive central hemodynamic monitors tend to have far more fluid administered without commensurate improvement in outcome. While invasive monitoring may be indicated for patients with co-morbidities or patients who fail to respond to resuscitation prescriptions, a blanket endorsement of this approach cannot be made. Third, antioxidant therapies show promise in reduction of burn resuscitation fluid requirements and edema formation in a variety of preclinical trials. Unfortunately, patient data is limited and multicenter prospective validation has not been attempted.

\section{Sepsis and Infection in Burn-Injured} Patients

In an important action, the ABA convened a consensus conference addressing burn sepsis and infection using methodology recently employed by the Society of Critical Care Medicine and other critical care societies [6]. The conference and the documents produced from it applied consensus-driven definitions of organ dysfunction and infection as described in the general critical care population and modified these as appropriate to reflect the perturbations encountered in burn injury. This work is important, as the major cause of late death in the burn patient population is multiple organ dysfunction syndrome, which typically is driven by infection. Findings of this consensus conference process are highlighted below.

The concept of Systemic Inflammatory Response Syndrome (SIRS) should not be applied to burn patients [10]. While the SIRS concept has been widely accepted and utilized in critical care practice and clinical trials, it has been widely criticized for being too inclusive and insufficiently specific to effectively identify a relevant inflammatory state. Burn patients frequently demonstrate characteristics of SIRS throughout the majority of hospitalization. Biochemical markers have also been evaluated but at present do not apply to the specific physiology of the burned patient.

Sepsis should be redefined for the burn patient population [6]. Triggers in the burn-injured patient are different than those in other critical care populations. As in general critical care practice, sepsis is a condition warranting empiric antibiotics and a search for infection during that short course of empiric therapy.

The concept of severe sepsis, the intervening state between sepsis and septic shock was dropped as the conference attendees felt that a distinctly separate state between sepsis and septic shock is not regularly seen. Septic shock definitions from consensus conferences including the Surviving Sepsis Campaign and consensus work of the major critical care societies are retained [11]. Of note, the ABA has not yet addressed the updated definition system reflected in the recent Sepsis-3 Consensus Statement [12].

Septic shock for the burned patient is defined as sepsisinduced hypotension that persists despite adequate fluid 
resuscitation. Sepsis-induced hypotension is defined as a systolic blood pressure (SBP) $<90 \mathrm{mmHg}$ or mean arterial pressure $<70 \mathrm{mmHg}$ or a SBP decrease $>40 \mathrm{mmHg}$ or $>2 \mathrm{SD}$ below normal for age in the absence of other identified causes of hypotension. Sepsis-induced tissue hypoperfusion is defined as septic shock, an elevated lactate, or oliguria [6].

Smoke inhalation injury by anatomic definition is inflammation occurring below the glottis that results from exposure to the by-products of combustion. Diagnosis requires a relevant history of acute exposure and bronchoscopy that reveals carbonaceous material or signs of edema/ulceration. Smoke inhalation injury can occur with or without detection of products such as cyanide or carbon monoxide. Bronchoscopy demonstrating anatomic injury is the "gold standard" for diagnosis.

Pneumonia, a common complication of inhalation injury, is defined in a fashion similar to those of previous consensus conferences conducted by critical care and respiratory societies $[13,14]$. The ABA Consensus Group did make statements regarding positive microbiology. Finding $\geq 10^{5}$ organisms on a tracheal aspirate, bronchoalveolar lavage with $\geq 10^{4}$ organisms, and protected bronchial brushings with $>10^{3}$ organisms per $\mathrm{mL}$ are criteria for positive microbiology in the setting of burn injury. The burn literature supports discontinuation of antibiotics when these microbiologic thresholds are not met. The Clinical Pulmonary Infection Score was briefly discussed and felt to be insufficient to predict VentilatorAssociated Pneumonia (VAP) in the burn victim. Clinical suspicion of ventilator-associated pneumonia must be verified by quantitative culture results.

VAP is an important complication in burn-injured patients. Burn patients are at high risk for developing VAP, particularly in the setting of inhalation injury. Prevention strategies are recommended, but relatively few are supported by strong evidence. Clinical diagnosis of VAP can be challenging in mechanically ventilated burn patients, a population in whom systemic inflammation, acute lung injury and non-infectious pulmonary infiltrates are prevalent. Quantitative strategies for identifying pathogens are the preferred method to confirm the diagnosis of VAP. Empiric antimicrobial therapy should cover resistant Staphylococcus aureus and gram-negative bacilli. In general, at least eight days of antibiotic therapy are recommended [15].

Several therapies are still under investigation to optimize outcome of VAP in burn-injured patients. Perhaps the most discussed is protocolized management of smoke inhalation. A variety of ventilation modes have been recommended. A recent survey of ventilator practice conducted by burn centers reveals a wide range of behavior. In addition, the role of early tracheostomy to facilitate secretion clearance remains unclear, along with the optimal means to deliver antimicrobials. While most centers use intravenous administration, several investigators are assessing the value of aerosolized antibiotics, which could enhance delivery to the lower respiratory tract. An encouraging development comes from the Division of Burn Surgery at the University of California-Davis, who introduced a VAP prevention bundle including elevation of the head-of-the-bed to 30 degrees, daily oral care with chlorhexidine, daily sedation interruption with assessment of readiness to wean from mechanical ventilation, and prophylaxis against stress ulceration and deep venous thrombosis. Compliance with the bundle was facilitated by a nurse advocate dedicated to the intensive care unit. Not surprisingly, TBSA burn extent and number of ventilator days were independent predictors of development of VAP. However, data obtained after implementation of the management bundle revealed a significantly reduced development of VAP, independent of TBSA burn extent and number of ventilator days [16,17].

Definitions for bloodstream and catheter-related bloodstream infections are accepted as defined in previous reports [6]. Catheter colonization is indicated by growth of organisms from a catheter segment, as identified by semiquantitative or quantitative culture. A catheterrelated bloodstream infection reflects identification of the identical organism from blood culture and semiquantitative or quantitative culture from a catheter segment. Clinical symptoms of bloodstream infection should also 
be present without any other apparent source of infection. Finally, exit site infection is defined as erythema, tenderness, induration, or purulence within $2 \mathrm{~cm}$ of the exit site of a catheter. Blood cultures should ideally include quantitative techniques with a specimen of $\geq 10 \mathrm{~mL}$. Blood cultures must also reflect recognized pathogens not usually regarded as skin contaminants.

Definitions of wound-related complications were also assembled $[6,18]$. Wound colonization is present with bacteria on the wound surface at low concentrations. Wound infection is present with high concentrations of bacteria in the wound and wound eschar $\left(>10^{5}\right.$ bacteria/ gram tissue). Invasive infection occurs with high concentrations of pathogens ( $>10^{5}$ bacteria/gram tissue) and changes such as separation of eschar or skin grafts, invasion of adjacent unburned tissue or development of sepsis, as defined above. A diagnosis of cellulitis requires bacteria recovered from the wound or wound eschar in high concentrations and advancing erythema, induration, warmth, and tenderness of surrounding tissues. Sepsis, as defined above, must be present. Necrotizing fasciitis is an aggressive, invasive infection with necrosis of tissues beneath the skin. Objective diagnostic criteria include biopsy and swab culture techniques, but none of these is ideal. Clinical correlates include systemic changes compatible with serious infection, premature separation of eschar, and unexplained tissue loss or change in the depth of wounds. Pseudomonas aeruginosa is a frequent colonizing organism in burn and other soft tissue wounds. The yellow/green exudate of Pseudomonas does not necessarily indicate invasive infection. When changes consistent with deep tissue injury and systemic changes including organ dysfunction are seen, aggressive antibiotic therapy and surgical debridement are urgently warranted.

\section{Electrical and Lightning Injury}

An electrical injury occurs when a person comes in contact with the current produced by an electrical energy source. This source may be manmade, such as a power line, or a natural one, such as a lightning strike. Electrical injury that emanates from a manmade source is typically transmitted after its generation at very high voltage, but transformers reduce voltage gradually and power lines distributing electricity for homes, buildings, and most industries carry low voltage (typically less than 600 volts). Homes and buildings generally have a system providing $<240$ volts for general use. Therefore, relatively low voltage sources account for the majority of accidental injuries secondary to electricity [8].

Electric current exists in two forms, alternating current (AC) and direct current (DC). In AC, electrons flow back and forth through a conductor in cyclic fashion. This type of current is most commonly used in households and offices and is standardized to a frequency of 50 or 60 cycles per second, depending on country. When current is direct, the electrons flow in only one direction. This type of current is produced by various batteries and is used in medical equipment such as defibrillators, pacemakers, and electric scalpels. Although AC is considered to be a far more efficient way of generating and distributing electricity, it is more dangerous than DC because it causes tetanic muscle contractions that often prolong contact of the victim with the source $[8,19]$.

Lightning is a form of direct current generated when an electrical potential difference between a thunder cloud and the ground overcomes the insulating properties of the surrounding air. The current flow of a lightning strike rises to a peak in approximately 2 microseconds and it lasts only 1 to 2 milliseconds. Voltage of a lightning strike may exceed $1,000,000$ volts with greater than 200,000 amperes of current flow. Transformation of the electrical energy to heat can generate temperatures as high as $50,000^{\circ} \mathrm{F}$. However, the extremely short duration of lightning strikes prevents the struck object from melting in most cases [20].

In general, the type and extent of an electrical injury depends on the intensity of the electric current. According to Ohm's Law, electric current is proportional to the voltage of the source and inversely proportional to the 
resistance of the conductor. Because resistance varies significantly between tissues, exposure of different parts of the body to the same current will produce different conduction paths and result in different degrees of injury. The least resistance is found in the nervous system, blood, mucus membranes, and muscles; the highest resistance is found in bones, fat, and tendons. Skin has intermediate resistance. The skin is the primary resistor against electrical current with a resistance ranging in adults between 40,000 and 100,000 ohms depending on its thickness (thicker skin means higher resistance). Even more important than thickness of the skin in determining injury is the presence or absence of moisture on the skin. The presence of simple perspiration may decrease the resistance of the skin to less than $1,000 \mathrm{ohms}$. Wet skin in a bathtub or swimming pool may facilitate electrocution as it offers almost no resistance at all, generating the maximum intensity of current that the applied voltage can drive [8].

Duration of contact with electrical current is another important determinant of injury. An electric shock caused by alternating current will produce greater injury than a shock caused by direct current of the same amperage because direct current causes a single muscle contraction that tends to throw the victim away from the power source, whereas alternating current repeatedly stimulates muscle contraction, often trapping the victim into sustained contact with the energy source. The pathway of current through the body from entry to exit point determines the number of organs that are affected, and as a result, the type and severity of injury. Determination of the electrical pathway is important for acute management and for overall prognosis. For example, a vertical pathway parallel to the axis of the body is the most dangerous because it involves virtually all vital organs including the central nervous system, heart, respiratory muscles, and in the pregnant patient, the uterus, and fetus.

Electrical injury affects the cardiovascular system by directly causing necrosis of the cardiac muscle and by inciting dysrhythmias. The degree of myocardial injury depends on the voltage and type of current. Predisposi- tion to injury of the vascular bed reflects its high water content and excellent conductivity. Electric current affects vessels of different size in different ways. Large arteries are not immediately affected because their rapid blood flow allows for rapid heat dissipation. However these same large vessels are susceptible to medial necrosis and later sequelae such as aneurysm formation and the potential for delayed rupture. Smaller vessels are acutely affected due to coagulation necrosis, which may lead to ischemia and compartment syndromes. The most frequently encountered rhythm disturbance is sinus tachycardia, often accompanied by non-specific ST- and T-wave changes. Conduction defects such as heart blocks are also common [8,19-21]. Cardiac standstill and ventricular fibrillation are the most serious cardiac complications of electrical injury and are invariably fatal unless the victim is immediately resuscitated from them.

No pathognomonic change characterizes passage of electrical current through the nervous system. Acute cranial nerve deficits and seizures may occur after electrical injury to the brain. Direct injury to the spinal cord at the lower cervical level has also been reported. Loss of consciousness, confusion, and impaired recall are common among victims of electrical injury. If there is no associated tissue damage, these patients tend to recover well. Dysfunction of peripheral motor and sensory nerves may cause a variety of deficits. The most serious consequence of neural electrical injury is malfunction of the respiratory control center that culminates in sustained respiratory arrest. Other patients may exhibit a variety of additional non-specific abnormal respiratory patterns after electrical encounters $[8,22]$. There are no specific injuries to the lungs or airways directly associated with electric current.

Exposure to current generated by low voltage sources may cause cutaneous injury by transformation of electrical to thermal energy. Injuries can range from local erythema to full-thickness burns. As with other organs, the severity of a surface burn depends on the intensity of the current, surface involved, and duration of exposure. More serious burns are caused by exposure to arcs that are created in accidents with high voltage currents 
( $>1,000$ volts). In such cases, the severity of the burn depends not only on the temperature but also the energy within the arc. Burns due to lightning are common but typically quite superficial due to the short duration of contact between the energy source and the victim [19].

Management of electrical injury requires cardiopulmonary resuscitation and multiple organ system trauma care. Thorough evaluation for hidden injury, particularly to the spinal cord, should take place, including assessment for event-associated blunt thoracic or abdominal trauma. Imaging of the head, neck, chest, abdomen, and pelvis may be appropriate to rule out internal injury along with serial evaluation of liver, pancreatic, and renal function for traumatic and ischemic injury. Patients with high voltage injuries should be evaluated for rhabdomyolysis and heme-derived pigments in the urine. Limbs must be assessed for compartment syndromes that may require fasciotomy. Ophthalmologic and otoscopic evaluation may also be important [8].

It is not uncommon for electrical injuries, particularly those associated with lightning, to cause multiple casualties. In other instances of trauma that involve several simultaneously injured people, efforts are logically focused on those who show signs of life. Lightning victims are an exception to this general rule because patients struck by lightning may become acutely apneic due to paralysis of respiratory centers and have dilated nonreactive pupils due to autonomic dysfunction. However, it is possible for the heart to recover spontaneously. Thus, administration of oxygen and supplemental ventilation should be applied immediately to the apneic lightning victim as efforts are made for cardiac resuscitation. Airway control should be established as rapidly as possible to minimize the effects of anoxia, a major cause of mortality [20].

The combination of extensive burns and significant internal visceral injury that can accompany high voltage electrical exposures increases fluid requirements, due to extravasation into extra vascular compartments and ongoing fluid losses. Because muscle destruction that usually accompanies these injuries may cause myoglobinuria and lead to renal failure, aggressive fluid resuscitation is important. Whereas the appropriate fluid prescription for most burn patients can be determined by the extent of cutaneous injury, electrical and lightning injuries do not follow such rules, due to their higher incidence of internal injuries. Where any risk of cardiac injury is possible based on mechanism or energy source, cardiac monitoring with echocardiography to determine pump function is prudent. Enzyme markers of cardiac injury may be misleading, as normal enzyme concentrations in the circulating blood do not exclude the possibly of conducting system injury with consequent rhythm disturbances. This observation serves to emphasize the value of an interval monitoring $[8,19]$.

\section{Pulmonary Issues}

Respiratory failure in the burn victim is often characterized by hypoxemia with evolution to acute lung injury or acute respiratory distress syndrome (ARDS) [23]. Even in patients without defined inhalation injury, the presence of ARDS is associated with poorer outcome. Management of inhalation injury consists primarily of time-honored supportive care. This may include mechanical ventilation with supplemental oxygen therapy and effective pulmonary toilet aided by catheter means or bronchoscopic interventions.

Inhalation injury describes pulmonary trauma caused by inhalation of thermal or chemical irritants. Such injuries are classified into categories of heat injury (which is usually restricted to upper airway structures except in the case of steam jet exposure), of local chemical irritation to the respiratory tract, and of systemic toxicity that may occur with exposure to carbon monoxide or cyanide [24].

The classic description of the effects of inhalation injury and its principle complication, pneumonia, was produced in the 1980s from the U.S. Army Institute of Surgical Research. In a review of over 1,000 patients, subjects at risk for inhalation injury were investigated by bronchoscopy, Xenon lung scans, or both. Diagnosis of inhalation injury was made in 373 patients. With increas- 
ing burn size, there was a corresponding rise in incidence of inhalation injury. The diagnosis of pneumonia was made at approximately 10 days for patients with inhalation injury. Expected mortality rate was reported to rise from $20 \%$ in the presence of inhalation injury alone to $60 \%$ when both inhalation injury and pneumonia were present. Contributions of inhalation injury and pneumonia to mortality were found to be independent and additive. Expected mortality in patients with very small or very large burns was not affected by these pulmonary complications, except at the extremes of age.

While many techniques have been developed to manage cutaneous injury, relatively few diagnosis-specific therapies have been identified for the patient with inhalation injury. Improvements in mortality from inhalation injury are mainly attributed to general improvements in critical care rather than to focused interventions for smoke inhalation. A variety of factors explain slower progress for improvement in management of inhalation injury. Cutaneous burn injury may be excised and replaced with skin grafts, but injury to pulmonary tissue must merely be supported and protected from secondary injury. In addition to smoke inhalation, the critically ill burn patient often has multiple mechanisms contributing to lung injury, such as sepsis, ventilator-induced lung injury, or systemic inflammation in response to burns. Thus, inhalation injury impacts burn patient outcome but its role is difficult to separate from the contributions of other injury drivers which affect the lungs. Another significant limitation for clinicians treating smoke inhalation has been the lack of uniform criteria for diagnosis and scaling of outcome severity. As a result, multicenter trials are confounded by differing local definitions of inhalation injury. The need for widely accepted diagnostic criteria and for a quantifying system for inhalation injury has been acknowledged in the burn literature [24].

A large survey regarding mechanical ventilation practices was recently conducted in North American burn centers. Pressure support and volume assist control were most common initial mechanical ventilation modes used, regardless of the presence of smoke inhalation. Using the recent Berlin definition for ARDS, the ARDSNet protocols for patients treated positive end-expiratory pressure were the most popular ventilation choices along with fluid restriction and diuresis. For severe ARDS, airway pressure release ventilation (APRV) and neuromuscular blockade were commonly used. The most frequently reported timeframe for placement of tracheostomy was two weeks. In general, wide variation in clinical practice was noted among North American burn centers, with no single ventilator mode or treatment adjunct pre-eminent in the management of burn patients, regardless of pulmonary insult [17].

\section{Outcomes}

For over half a century, investigators have sought reliable predictive indices for outcomes from burn injury. Perhaps the best known is the Baux rule, a simple sum of patient age and total body surface area in those suffering 2 nd and 3 rd degree burns. This index continues to receive attention; in fact, the Baux rule was recently addressed using patient registry data from the ABA [25].

The most recent summary of outcomes in burn care comes from the 2016 National Burn Repository Annual Report produced by the ABA [26]. Relevant data is drawn from burn admissions during the time interval spanning 2006 to 2015. A number of important observations can be made. First, over 200,000 records are reviewed from a representative cross-section of U.S. burn centers. In all age categories, except age $>80$ years old, males outnumber females. Pediatric patients ranging in age from $1-15$ years comprised $30 \%$ of the total sample while patients 60 years or older represented $14 \%$ of burn cases reported.

More than $75 \%$ of all burns were $<10 \%$ TBSA, and these cases had a mortality rate of only $0.6 \%$. The mortality rates for all burns and for fire/flame injuries were $3.3 \%$ and $5.8 \%$, respectively. The two most common etiologies of burn injury were fire/flame and scalds, accounting for $75 \%$ of cases reported. Scald injuries were 
most prevalent in children under the age of 5 years while fire/flame injuries dominated in the remaining age categories. Seventy-three percent of burn injuries with a known place of event were reported to have occurred in the home. Nearly $95 \%$ of cases with known circumstances of injury were identified as accidents, with nearly $14 \%$ of burns reported as work-related. Just over $2 \%$ of cases were suspected abuse and $1 \%$ of burn injuries were selfinflicted.

During the 10 year period from 2006 to 2015 , the average length of stay for females declined from 9.3 days to 7.9 days while that for males declined less significantly, from 9.1 to 8.8 days. The mortality rate for females declined from $4.1 \%$ to $2.9 \%$ compared with $3.9 \%$ to $3 \%$ for males. Deaths from burn injury increased with advancing age, increasing burn size, and the presence of inhalation injury.

Pneumonia was the most frequent complication reported and occurred in $5.4 \%$ of fire/flame-injured patients. The frequency of pneumonia and respiratory failure was much greater in patients treated 4 days or more with mechanical ventilation. As age advances, the rate of complications increases (with the exception of infants who have a higher rate than other children).

Extensive burn wounds heal slowly and the period of critical illness tends to be protracted. For burn survivors, the average length of hospital stay was slightly greater than 1 day per \% TBSA burned. For patients who died, the total hospital days were nearly two times that of survivors on average; however, this trend was reversed in patients with $>20 \%$ TBSA burns. Eight-seven percent of patients were discharged to home and $3 \%$ of patients were transferred to rehabilitation facilities.

Overall, the charges for patients who died were more than three times greater than those for patients who survived; however, this was skewed by the relatively high proportion of the patient sample with burns $<10 \%$ TBSA. For burns $>10 \%$ TBSA treated in American hospitals, total charges for surviving patients averaged over $\$ 250,000$ U.S. and charges for non-survivors averaged $\$ 340,000$ U.S. [26].
A classic single center outcome review of over 1,600 patients admitted to the Massachusetts General Hospital and the Schriners' Burn Institute in Boston was published in early 1998 [27]. Logistic regression analysis was employed to develop probability estimates for mortality based on a small set of well-defined variables. Three risk factors for death were identified: age $>60$ years; TBSA burns $>40 \%$ and inhalation injury. The mortality formula developed from this report predicts $0.3 \%, 3 \%, 33 \%$ or $90 \%$ mortality depending on whether $0,1,2$ or 3 risk factors are present respectively.

Early recognition of organ dysfunction and sepsis may prevent further complications in the critically ill burned patient. Markers for renal injury have been investigated as a means to provide earlier recognition of acute renal failure [28]. Cystatin C, plasma neutrophil gelatinaseassociated lipocalin (NGAL), and urine NGAL were evaluated in patients demonstrating acute kidney injury within five days of burn injury. Mortality was high in both the early acute kidney injury patients (77\%) and patients with late presentation of acute kidney injury (58\%). NGAL was elevated within hours after injury in patients with early acute kidney injury and early death. Cystatin $\mathrm{C}$ and serum creatinine did not rise until 12 hours after injury.

Many severely injured patients are vulnerable to sepsis from many sources. Early diagnosis and treatment of such infections may reduce the incidence of complications and improve survival. Burn patients have been evaluated for effectiveness of N-terminal pro B-type natriuretic peptide (pro-BNP), procalcitonin, and hemodynamic monitors using arterial pressure waveform analysis to identify the presentation of sepsis [29]. ProBNP, systemic vascular resistance, and stroke volume variation appeared to have good predictive quality for identification of sepsis in burn patients. The combination of elevated pro-BNP and stroke volume variation with low systemic vascular resistance may help provide early identification of infectious complications.

Development of burn centers in the United States has been associated with a verification process to standardize 
and optimize overall quality of care delivered [30,31]. Perhaps as a direct beneficial consequence, burn centers have been demonstrated to improve survival and decrease resource consumption. Using the discharge database from the state of California, five burn centers verified by the ABA and the American College of Surgeons were compared with 12 non-verified burn centers. In 2003, 2,867 patients were admitted to burn centers; over 1,600 of these patients were admitted to non-verified centers. Verified centers admitted more patients with large burns, burns requiring complex reconstruction, and more patients requiring critical care support including mechanical ventilation. Verified burn centers also performed fewer operations than non-verified centers. More patients from verified centers were able to return home while additional rehabilitation care was required in patients from non-verified centers. Mortality, however, was $3 \%$ in non-verified burn centers and $4 \%$ in verified burn centers, perhaps in part reflecting the severity mix. Differences in resource consumption including operative procedures, post-discharge destination, and median hospital charge support the use of verified burn centers in the management of burn-injured patients. Clearly, the population seen in verified and non-verified burn centers is different and this report details resource consumption issues which may be better addressed in a regionalized burn care system [32].

\section{Conclusions}

The burn patient is easily over resuscitated. Practitioners must be willing to reduce fluid prescriptions when signs of adequate perfusion are present. Currently, adequate vital signs and urine output are the "gold standard" for perfusion assessment.

In the burn-injured patient traditional definitions of systemic inflammatory response syndrome (SIRS) and sepsis must be redefined based on the physiologic characteristics of burn injury. In addition, the burn patient is at risk for soft tissue infections and burn wound infection which have been better defined.

Inhalation injury that requires respiratory assistance should be provided in accordance with the same principles of lung protective mechanical ventilation used in other patients with ARDS.

Upper airway damage and smoke inhalation injury can occur with or without detection of oropharyngeal change or detection of combustion products in the blood such as cyanide or carbon monoxide. Bronchoscopy demonstrating anatomic injury represents the "gold standard" for diagnosis.

A number of factors predict mortality in burn injury. Burn size, presence or absence of inhalation injury, and extremes of age have been widely reported to be influential.

Renal failure and insufficiency are strongly associated with poor outcome following extensive burns.

Among the special problems addressed in burn centers, electrical injuries pose multisystem physiologic challenges and do not fit typical scoring systems based on abnormalities observed at the skin surface.

Management by verified burn centers reduces cost of therapy for burn injury.

\section{Acknowledgements}

The authors gratefully recognize the technical assistance of Mrs. Sherry Willett in the preparation of this manuscript.

\section{ORCID}

David J. Dries ～http://orcid.org/0000-0002-4366-6318

\section{References}

1. Latenser BA. Critical care of the burn patient: the first 48 hours. Crit Care Med 2009; 37: 2819-26.

2. Baxter CR. Fluid volume and electrolyte changes of the early postburn period. Clin Plast Surg 1974; 1: 
693-703.

3. Saffle JR. The phenomenon of "fluid creep" in acute burn resuscitation. J Burn Care Res 2007; 28: 382-95.

4. Pham TN, Cancio LC, Gibran NS; American Burn Association. American Burn Association practice guidelines burn shock resuscitation. J Burn Care Res 2008; 29: 257-66.

5. Blumetti J, Hunt JL, Arnoldo BD, Parks JK, Purdue GF. The Parkland formula under fire: is the criticism justified? J Burn Care Res 2008; 29: 180-6.

6. Greenhalgh DG, Saffle JR, Holmes JH 4th, Gamelli RL, Palmieri TL, Horton JW, et al. American Burn Association consensus conference to define sepsis and infection in burns. J Burn Care Res 2007; 28: 776-90.

7. Uchino S, Kellum JA, Bellomo R, Doig GS, Morimatsu H, Morgera S, et al. Acute renal failure in critically ill patients: a multinational, multicenter study. JAMA 2005; 294; 813-8.

8. Koumbourlis AC. Electrical injuries. Crit Care Med 2002; 30(11 Suppl): S424-30.

9. Ivy ME, Atweh NA, Palmer J, Possenti PP, Pineau M, D’Aiuto M. Intra-abdominal hypertension and abdominal compartment syndrome in burn patients. J Trauma 2000; 49: 387-91.

10. Bone RC, Balk RA, Cerra FB, Dellinger RP, Fein AM, Knaus WA, et al. Definitions for sepsis and organ failure and guidelines for the use of innovative therapies in sepsis. The ACCP/SCCM Consensus Conference Committee. American College of Chest Physicians/Society of Critical Care Medicine. Chest 1992; 101: 1644-55.

11. Dellinger RP, Levy MM, Rhodes A, Annane D, Gerlach H, Opal SM, et al. Surviving sepsis guidelines: international guidelines for management of severe sepsis and septic shock: 2012. Crit Care Med 2013; 41: 580-637.

12. Singer M, Deutschman CS, Seymour CW, ShankarHari M, Annane D, Bauer M, et al. The third international consensus definitions for sepsis and septic shock (Sepsis-3). JAMA 2016; 315: 801-10.
13. Hubmayr RD, Burchardi H, Elliot M, Fessler H, Georgopoulos D, Jubran A, et al. Statement of the 4th international consensus conference in critical care on ICU-acquired pneumonia--Chicago, Illinois, May 2002. Intensive Care Med 2002; 28: 1521-36.

14. Rello J, Paiva JA, Baraibar J, Barcenilla F, Bodi M, Castander D, et al. International conference for the development of consensus on the diagnosis and treatment of ventilator-associated pneumonia. Chest 2001; 120: 955-70.

15. Mosier MJ, Pham TN. American Burn Association practice guidelines for prevention, diagnosis, and treatment of ventilator-associated pneumonia (VAP) in burn patients. J Burn Care Res 2009; 30: 910-28.

16. Sen S, Johnston C, Greenhalgh D, Palmieri T. Ventilator-associated pneumonia prevention bundle significantly reduces the risk of ventilator-associated pneumonia in critically ill burn patients. J Burn Care Res 2016; 37: 166-71.

17. Chung KK, Rhie RY, Lundy JB, Cartotto R, Henderson E, Pressman MA, et al. A survey of mechanical ventilator practices across burn centes in North America. J Burn Care Res 2016; 37: e131-9.

18. Peck MD, Weber J, McManus A, Sheridan R, Heimbach D. Surveillance of burn wound infections: a proposal for definitions. J Burn Care Rehabil 1998; 19: 386-9.

19. Arnoldo BD, Purdue GF, Kowalske K, Helm PA, Burris A, Hunt JL. Electrical injuries: a 20-year review. J Burn Care Rehabil 2004; 25: 479-84.

20. Lichtenberg R, Dries D, Ward K, Marshall W, Scanlon P. Cardiovascular effects of lightning strikes. J Am Coll Cardiol 1993; 21: 531-6.

21. Arnoldo B, Klein M, Gibran NS. Practice guidelines for the management of electrical injuries. J Burn Care Res 2006; 27: 439-47.

22. Singerman J, Gomez M, Fish JS. Long-term sequelae of low-voltage electrical injury. J Burn Care Res 2008; 29: 773-7.

23. ARDS Definition Task Force, Ranieri VM, Rubenfeld GD, Thompson BT, Ferguson ND, Caldwell E, 
et al. Acute respiratory distress syndrome: the Berlin Definition. JAMA 2012; 307: 2526-33.

24. Dries DJ, Endorf FW. Inhalation injury: epidemiology, pathology, treatment strategies. Scand J Trauma Resusc Emerg Med 2013; 21:31.

25. Jeng JC. Patrimonie de Docteur Baux--Baux Scores >> 100 gleaned from 170,791 admissions: a glimmer from the National Burn repository. J Burn Care Res 2007; 28: 380-1.

26. American Burn Association. 2016 National Burn Repository. Report of data from 2006-2015 [Internet]. Chicago, American Burn Association; c2017. [cited 2017 Jan 12]. Available from: http://www.ameriburn. org/NBR.php.

27. Ryan CM, Schoenfeld DA, Thorpe WP, Sheridan RL, Cassem EH, Tompkins RG. Objective estimates of the probability of death from burn injuries. N Engl J Med 1998; 338: 362-6.

28. Paratz JD, Lipman J, Boots RJ, Muller MJ, Paterson
DL. A new marker for sepsis post burn injury?* Crit Care Med 2014; 42: 2029-36.

29. Yang HT, Yim H, Cho YS, Kym D, Hur J, Kim JH, et al. Assessment of biochemical markers in the early post-burn period for predicting acute kidney injury and mortality inpatients with major burn injury: comparison of serum creatinine, serum cystati$\mathrm{C}$, plasma and urine neutrophil gelatinase-associated lipocalin. Crit Care 2014; 18: R151.

30. Palmieri TL, London JA, O'Mara MS, Greenhalgh DG. Analysis of admissions and outcomes in verified and nonverified burn centers. J Burn Care Res 2008; 29: 208-12.

31. Ehrlich PF, Rockwell S, Kincaid S, Mucha P Jr. American College of Surgeons, Committee on Trauma Verification Review: does it really make a difference? J Trauma 2002; 53: 811-6.

32. Sheridan RL. Burn care: results of technical and organizational progress. JAMA 2003; 290: 719-22. 